\title{
Carbohydrate quality is not associated with liver enzyme activity and plasma TAG and HDL concentrations over 5 years in an older population
}

\author{
Janina Goletzke ${ }^{1}$, Anette E. Buyken ${ }^{1 *}$, Bamini Gopinath ${ }^{2}$, Elena Rochtchina ${ }^{2}$, Alan W. Barclay ${ }^{3}$, \\ Guo Cheng ${ }^{4,5,6}$, Jennie C. Brand-Miller ${ }^{3}$ and Paul Mitchell ${ }^{2}$ \\ ${ }^{1}$ IEL-Nutritional Epidemiology, DONALD Study at the Research Institute of Child Nutrition, University of Bonn, \\ 44225 Dortmund, Germany \\ ${ }^{2}$ Department of Ophthalmology, Centre for Vision Research, Westmead Millennium Institute, University of Sydney, \\ Sydney, NSW 2006, Australia \\ ${ }^{3}$ Department of Molecular and Microbial Biosciences, Boden Institute of Obesity, Nutrition and Exercise, \\ University of Sydney, Sydney, NSW 2006, Australia \\ ${ }^{4}$ Fulda University of Applied Sciences, Fulda, Germany \\ ${ }^{5}$ West China School of Public Health, Sichuan University, Chengdu, People's Republic of China \\ ${ }^{6}$ Food Safety Monitoring and Risk Assessment Key Laboratory of Sichuan Province, Sichuan University, \\ Chengdu, People's Republic of China
}

(Submitted 20 July 2012 - Final revision received 4 December 2012 - Accepted 4 December 2012 - First published online 23 January 2013)

\begin{abstract}
Non-alcoholic fatty liver disease (NAFLD) is closely associated with insulin resistance and obesity. Hence, carbohydrate quality could be of relevance to the risk of NAFLD, but prospective data are lacking. The aim of the present study was to investigate longitudinal associations between carbohydrate quality (including dietary glycaemic index (GI) and intakes of sugar, starch and fibre) and markers of liver function in an older Australian population. The analysis was based on 866 participants ( $\geq 49$ years) of the Blue Mountains Eye Study with fasting blood specimens and dietary intake data at baseline and 5-year follow-up. Multi-level mixed regression analysis was used to relate dietary GI and sugar, starch and fibre intake to the liver enzymes alanine aminotransferase (ALT) and $\boldsymbol{\gamma}$-glutamyltransferase (GGT), as well as fasting TAG and HDL-cholesterol (HDL-C). After adjustment for potential confounding factors, a lower fibre intake was cross-sectionally related to higher GGT $(P=0.02)$ and fasting TAG $(P=0.002)$ levels, with fruit fibre being the most relevant fibre source $(P=0.095$ for GGT; $P=0.003$ for TAG). A higher dietary GI was associated with lower HDL-C $(P=0.046)$. Changes in carbohydrate quality during 5 years were not related to changes in ALT, GGT, TAG or HDL-C $(P \geq 0.08)$. In conclusion, the absence of longitudinal associations between carbohydrate quality and liver enzymes and serum lipids in this older population does not support a major role of carbohydrate nutrition in liver function among the elderly.
\end{abstract}

Key words: Non-alcoholic fatty liver disease: Serum lipids: Glycaemic index: Dietary fibre

Non-alcoholic fatty liver disease (NAFLD) describes a condition of fat accumulation in hepatocytes in the absence of other causes of hepatic steatosis such as excess alcohol consumption. Recently, NAFLD has been recognised as an important risk factor for the development of both type 2 diabetes and CHD. In this context, NAFLD has also been termed as the hepatic manifestation of the metabolic syndrome, as it has been related to all its constituting features ${ }^{(1)}$.

The close relationship with obesity and insulin resistance suggests a link to dietary factors associated with disturbances in glucose and insulin metabolism. Indeed, several recent cross-sectional $^{(2,3)}$ and case-control studies ${ }^{(4,5)}$ have reported associations between carbohydrate quality and NAFLD risk in adults aged 50-60 years ${ }^{(2,3,5)}$ as well as younger adults aged 30 years $^{(4)}$. Both higher dietary glycaemic index $(\mathrm{GI})^{(2)}$ (which ranks carbohydrates according to their glycaemic potency) and added sugars ${ }^{(4,5)}$, particularly from soft drinks, were related to increased NAFLD markers. By contrast, studies on the relevance of dietary fibre for liver fat content have yielded inconsistent results in middle-aged ${ }^{(6,7)}$ and older adults ${ }^{(2)}$. Overall, evidence from prospective studies is lacking, but would be of importance to shed light on the longitudinal

Abbreviations: ALT, alanine aminotransferase; BMES, Blue Mountains Eye Study; GGT, $\gamma$-glutamyltransferase; GI, glycaemic index; HDL-C, HDL-cholesterol; NAFLD, non-alcoholic fatty liver disease.

*Corresponding author: A. E. Buyken, fax +49 2317115 81, email buyken@fke-do.de 
relevance of carbohydrate quality for NAFLD risk. As the prevalence of NAFLD increases with age ${ }^{(1)}$, investigating this relationship in older cohorts is relevant.

The liver enzymes alanine aminotransferase (ALT) and $\boldsymbol{\gamma}$-glutamyltransferase (GGT) are commonly used as noninvasive estimates for hepatic fat accumulation, but in contrast to ALT, GGT also serves as a marker for alcoholic fatty liver ${ }^{(1,8-10)}$. Serum lipids can be considered additionally as risk markers, since NAFLD is closely related to dyslipidaemia and the metabolic syndrome ${ }^{(11,12)}$. Elevated hepatic lipogenesis leads to an overproduction of TAG and subsequently VLDL; hence, increased levels of fasting TAG may indicate metabolic alterations in the liver ${ }^{(13)}$. Low HDL-cholesterol (HDL-C) values serve as a further marker for disturbances in lipid metabolism, and, indeed, a study of non-obese, non-diabetic subjects showed that, among others, hypertriacylglycerolaemia and low levels of HDL-C were risk factors for NAFLD ${ }^{(14)}$.

The present study aims to examine both the cross-sectional and the 5-year concurrent associations between carbohydrate quality (GI and intake of sugar, starch and fibre) and the proposed markers of liver function (ALT, GGT, fasting TAG and HDL-C) in an older population.

\section{Materials and methods}

\section{Study population}

The Blue Mountains Eye Study (BMES) is a population-based cohort study of vision, common eye diseases and other health outcomes in an urban, predominantly Caucasian Australian population aged 49 years and older, which was initiated in 1992 with two 5-year follow-up examinations. A total of 3654 participants took part in the baseline examination (1992-4; BMES-1 $)^{(15)}$. The study was conducted in accordance with the recommendations of the Helsinki Declaration and was approved by the University of Sydney and the Sydney West Area Health Service Human Research Ethics Committees. Written informed consent was obtained from all participants. Details on the study and covariate assessment have been described elsewhere ${ }^{(15)}$.

\section{Study sample}

We were interested to examine the cross-sectional and the 5-year concurrent associations of dietary carbohydrate quality with liver enzymes, fasting TAG and HDL-C concentrations. Liver enzymes were first analysed in the first follow-up (BMES-2). Hence, in the present analysis, these data were termed baseline and BMES-3 data (second re-examination) as the respective 5-year follow-up. The analysis was restricted to the participants who had provided a complete and plausible FFQ, as well as a fasting blood specimen at both BMES-2 and -3.

Of the 2335 participants re-examined at BMES-2, 330 had to be excluded due to incomplete ( $\geq 12$ questions of the FFQ missing, an entire page remaining blank) or implausible dietary data (daily energy intakes were $<2500$ or $>18000 \mathrm{~kJ}^{(16)}$ ), and a further 195 because of missing blood samples. Of the remaining 1810 participants, 388 died during the 5-year follow-up period, 161 were lost to follow-up and 218 participated in BMES-3 but did not provide a plausible FFQ and/or a blood sample. A total of 1043 participants had dietary data and blood specimens at both BMES-2 and -3 . Another sixty-four participants were excluded because data on BMI, use of lipid-lowering drugs, diabetes mellitus, CHD or smoking status were missing. Moreover, to avoid any confounding due to alcoholic fatty liver disease, we excluded all BMES participants who consumed more than $20 \mathrm{~g}$ alcohol per $\mathrm{d}$ at both baseline and follow-up ( $n$ 113), a cut-off point which has commonly used ${ }^{(1,8)}$. Therefore, the present examination included 866 participants for the analysis of ALT and GGT. Furthermore, at BMES-3, some participants provided only nonfasting blood specimens; hence, fasting TAG and HDL-C concentrations from both visits were available for 755 participants only.

\section{Dietary assessment}

Dietary data were collected using a 145-item FFQ modified for the Australian diet and vernacular from an early Willett questionnaire $^{(17)}$. This FFQ was validated against $4 \mathrm{~d}$ weighed food records collected on three occasions during 1 year ( $n$ 79) and showed moderate-to-good agreement for ranking individuals according to their GI, dietary fibre and total carbohydrate intake ${ }^{(18)}$.

Nutrient intakes were estimated using the Australian Tables of Food Composition (NUTTAB95) and published GI values with the glucose $=100$ scale $^{(19)}$. Additional GI data were obtained from the Sydney University Glycaemic Index Research Service online database (www.glycaemicindex.com). An overall GI value for each participant's diet was calculated by summing the weighted GI of individual foods in the diet with the weighting proportional to the contribution of individual foods to total carbohydrate intake. Additionally, data on total fibre intake and fibre intakes from bread and cereals, vegetables and fruits were extracted from the FFQ.

\section{Liver enzymes and serum lipid levels}

Fasting blood specimens were drawn, centrifuged on site and then sent by courier within the same day to the Westmead Hospital, Sydney, for haematological analysis and clinical biochemistry assessment. Fasting serum TAG concentrations were measured on a Reflotron reflectance photometric analyser (Boehringer Mannheim Diagnostics; currently, Roche Diagnostics). CV for repeated measurements of plasma were $1 \cdot 4 \%$ for TAG and $3 \cdot 2 \%$ for HDL-C. ALT and GGT were determined using commercial kits performed on an automated analyser (OCD Fusion 5.1; Ortho Clinical), and CV were below $4 \%$ for ALT and below $2 \cdot 8 \%$ for GGT.

\section{Statistical methods}

Statistical analyses were performed using SAS software (version 9.1.3; SAS). Because some of the metabolic and nutritional data were not normally distributed, all continuous data are presented as medians (25th and 75th percentiles). 
Differences between baseline and the 5-year follow-up in metabolic variables and nutritional intake data were analysed using the Wilcoxon signed-rank test for continuous variables and the Mantel-Haenszel $\chi^{2}$ test for categorical variables.

We used linear mixed-effect regression models (PROC MIXED in SAS) to construct longitudinal models of trends in ALT, GGT, fasting TAG and HDL-C between baseline and the 5-year follow-up. Because the outcome variables were not normally distributed, all of them were log-transformed before the regression analysis.

In model 1, the following fixed effects were included: sex; time (defined as 1 (baseline) and 2 (5-year follow-up)); the respective dietary variable at baseline; the interaction of this dietary variable with time; the change in the dietary variable, calculated by subtracting baseline values of the respective parameter from the one at follow-up. In this way, the analysis yielded three regression coefficients representing the following: (1) the cross-sectional estimate - an estimate for the regression of carbohydrate quality at baseline on markers of hepatic fat accumulation at baseline; (2) the prospective estimate - the slope of the regression of carbohydrate quality at baseline on the change in the outcomes over 5 years; (3) the concurrent estimate - an estimate for the regression of the change in carbohydrate quality between the 5 years on the concurrent change in the outcomes. The parameters of carbohydrate quality (GI and intake of sugar, starch and fibre; total fibre and fibre from bread and cereals, vegetables and fruits) were energy-adjusted using the multivariate energy density model ${ }^{(20)}$, which required the calculation of fibre densities $(\mathrm{g} / \mathrm{MJ})$.

For model 2, the fixed effects of age, diabetes mellitus, any CHD, current or former smoking status, menopausal status, hormone replacement therapy, post-secondary school qualification, good self-rated health and use of cholesterol-lowering as well as other potential influencing medications, e.g. antidiabetic drugs, at baseline were considered as potential influencing factors. BMI and other metabolic and nutritional variables (e.g. total or saturated fat) were additionally examined, including their level at baseline, interaction of baseline level and time, or change in their level during the 5-year period. Only those potential influencing factors that (1) substantially modified the association of the principal dietary variables with ALT, GGT, TAG or HDL-C in the unadjusted models, (2) significantly predicted the outcome variable or (3) improved the fit statistic (Akaike's information criterion) were included in model 2. All analyses were performed with a significance level at $P<0.05$.

\section{Results}

Among the 866 participants included in the present analysis, more were women and individuals with a younger age, a post-secondary school qualification, a good self-reported health and overweight, but fewer suffered from CHD and smoked compared with the 831 BMES participants who had died or were lost to follow-up. Moreover, those included had lower ALT and fasting glucose concentrations and had consumed slightly more polyunsaturated fat, protein, carbohydrates, sugar, starch and fibre, especially from bread and cereals, and had a lower dietary GI at baseline (data not shown).

Baseline characteristics of the 866 BMES participants included in the present analysis are shown in Table 1. After the 5-year follow-up, the participants' BMI was lower and serum concentrations of ALT, GGT, albumin and bilirubin were higher, whereas concentrations of fasting TAG, glucose, HDL-C and cholesterol improved significantly (Table 2). Regarding 5-year changes in nutritional intake data, energy intake as well as total fat, and saturated fat intake were higher, whereas starch and fibre intake from bread and cereals were lower (Table 2). All of the observed changes were, however, very small.

\section{Carbohydrates and liver enzymes}

Carbohydrate quality was not independently associated with ALT, neither in the cross-sectional nor in the concurrent analysis (Table 3, first and second main columns).

The cross-sectional analysis showed inverse associations between fibre intake and GGT in model 1. These were attenuated, but still significant, after adjustment for potentially confounding factors $(P=0.02$, model 2 ; Table 3 , third main column). Among the fibre sources, fruit fibre seemed to have the greatest relevance for GGT levels, with higher intakes being related to lower GGT levels. However, this association was attenuated towards a trend after adjustment for confounding factors ( $P=0.095$, model 2 ; Table 3 , third main column). None of the other parameters of carbohydrate quality was related to GGT - neither in the cross-sectional nor in the concurrent change analyses.

\section{Carbohydrates and TAG and HDL-cholesterol}

Higher levels of dietary GI were associated with higher fasting TAG in the cross-sectional analysis, but this association was no longer evident after adjustment for confounding factors. Conversely, associations between fibre intakes and fasting TAG levels in the cross-sectional analysis were maintained ( $P=0.002$, model 2; Table 4, first main column), with higher

Table 1. Baseline characteristics of the 866 Blue Mountains Eye Study participants

(Percentages, or medians and 25th and 75th percentiles)

\begin{tabular}{|c|c|c|}
\hline & Total $(n)$ & Value (\%) \\
\hline \multicolumn{3}{|l|}{ Sex } \\
\hline Female & 866 & $62 \cdot 7$ \\
\hline Age (years) & 866 & \\
\hline Median & \multicolumn{2}{|c|}{$67 \cdot 0$} \\
\hline 25th percentile & \multicolumn{2}{|c|}{$62 \cdot 0$} \\
\hline 75th percentile & \multicolumn{2}{|c|}{$73 \cdot 0$} \\
\hline Diabetes mellitus & 866 & 9.8 \\
\hline Any CHD & 866 & $16 \cdot 3$ \\
\hline Cholesterol-lowering medication & 866 & $9 \cdot 2$ \\
\hline Menopause* & 543 & 94.3 \\
\hline Hormone replacement therapy, ever* & 543 & 38.7 \\
\hline Smoking & 866 & $41 \cdot 1$ \\
\hline Post-secondary school qualification & 835 & $64 \cdot 2$ \\
\hline Good self-reported health at baseline & 866 & 84.2 \\
\hline
\end{tabular}

* Percentage refers to women only. 
Table 2. Comparison of the metabolic variables and nutritional intake of the 866 Blue Mountains Eye Study participants at baseline and 5 -year follow-up

(Median values and 25th and 75th percentiles)

\begin{tabular}{|c|c|c|c|c|c|c|c|c|}
\hline & \multirow[b]{2}{*}{$n$} & \multicolumn{3}{|c|}{ Baseline } & \multicolumn{3}{|c|}{ 5-year follow-up } & \multirow[b]{2}{*}{$P^{*}$} \\
\hline & & Median & 25th percentile & 75th percentile & Median & 25th percentile & 75th percentile & \\
\hline \multicolumn{9}{|l|}{ Metabolic variables } \\
\hline $\operatorname{BMI}\left(\mathrm{kg} / \mathrm{m}^{2}\right)$ & 866 & $26 \cdot 8$ & $24 \cdot 4$ & $29 \cdot 9$ & $26 \cdot 6$ & $24 \cdot 1$ & $29 \cdot 8$ & $<0.0001$ \\
\hline $\operatorname{ALT}(U / I) \dagger$ & 866 & 19 & 15 & 26 & 22 & 18 & 28 & $<0.0001$ \\
\hline GGT $(U / I) \dagger$ & 866 & 20 & 15 & 29 & 23 & 17 & 32 & $<0.0001$ \\
\hline Albumin (\%) & 866 & 42 & 40 & 44 & 43 & 41 & 44 & $<0.0001$ \\
\hline Bilirubin, total $(\mu \mathrm{mol} / \mathrm{l})$ & 865 & 10 & 8 & 12 & 11 & 8 & 14 & $<0.0001$ \\
\hline Fasting glucose $(\mathrm{mmol} / \mathrm{l})$ & 737 & $5 \cdot 0$ & 4.7 & $5 \cdot 5$ & 4.9 & 4.6 & $5 \cdot 3$ & $<0.0001$ \\
\hline Fasting TAG (mmol/l) & 755 & 1.34 & 0.98 & 1.83 & $1 \cdot 28$ & 0.93 & $1 \cdot 75$ & 0.0001 \\
\hline Fasting cholesterol $(\mathrm{mmol} / \mathrm{l})$ & 866 & $6 \cdot 0$ & $5 \cdot 3$ & $6 \cdot 6$ & 5.4 & 4.8 & $6 \cdot 1$ & $<0.0001$ \\
\hline Fasting HDL (mmol/l) & 755 & 1.4 & $1 \cdot 2$ & $1 \cdot 7$ & 1.6 & $1 \cdot 3$ & 1.9 & $<0.0001$ \\
\hline \multicolumn{9}{|l|}{ Daily nutritional intakes } \\
\hline Energy (kJ) & 866 & 8201 & 6869 & 9750 & 8360 & 6890 & 10112 & 0.004 \\
\hline Fat $(\%)$ & 866 & 31.6 & $27 \cdot 8$ & $35 \cdot 6$ & 32.5 & 28.4 & 36.6 & $<0.0001$ \\
\hline SFA (\%) & 866 & $12 \cdot 1$ & $10 \cdot 1$ & $14 \cdot 2$ & $12 \cdot 5$ & $10 \cdot 4$ & 14.5 & 0.02 \\
\hline MUFA (\%) & 866 & $11 \cdot 3$ & $9 \cdot 9$ & $12 \cdot 8$ & 11.9 & $10 \cdot 2$ & 13.4 & $<0.0001$ \\
\hline PUFA (\%) & 866 & 4.9 & 3.9 & $6 \cdot 1$ & $5 \cdot 1$ & $4 \cdot 0$ & $6 \cdot 2$ & 0.05 \\
\hline Protein (\%) & 866 & $17 \cdot 6$ & $15 \cdot 7$ & $19 \cdot 6$ & $17 \cdot 7$ & $15 \cdot 8$ & $19 \cdot 7$ & 0.2 \\
\hline Carbohydrates (\%) & 866 & $47 \cdot 8$ & 43.5 & $52 \cdot 4$ & $47 \cdot 1$ & $42 \cdot 8$ & 51.4 & 0.002 \\
\hline Sugar (\%) & 866 & $25 \cdot 2$ & $21 \cdot 2$ & $29 \cdot 2$ & $25 \cdot 6$ & $21 \cdot 6$ & $29 \cdot 4$ & 0.1 \\
\hline Starch (\%) & 866 & $21 \cdot 7$ & $18 \cdot 7$ & $24 \cdot 9$ & $20 \cdot 5$ & $17 \cdot 4$ & $23 \cdot 2$ & $<0.0001$ \\
\hline GI & 866 & $56 \cdot 1$ & $53 \cdot 2$ & $58 \cdot 6$ & $56 \cdot 1$ & 53.4 & $58 \cdot 6$ & 0.6 \\
\hline $\mathrm{GL}$ & 866 & $132 \cdot 0$ & $106 \cdot 1$ & $158 \cdot 3$ & $132 \cdot 6$ & $106 \cdot 1$ & $160 \cdot 5$ & 0.5 \\
\hline Fibre $(g / 1000 \mathrm{~kJ})$ & 866 & 3.28 & $2 \cdot 71$ & 3.98 & 3.22 & $2 \cdot 65$ & 3.87 & 0.01 \\
\hline From vegetables $(\mathrm{g} / 1000 \mathrm{~kJ})$ & 866 & $1 \cdot 14$ & 0.90 & 1.47 & $1 \cdot 17$ & 0.93 & 1.51 & 0.05 \\
\hline From fruits $(\mathrm{g} / 1000 \mathrm{~kJ})$ & 866 & 0.78 & 0.49 & $1 \cdot 13$ & 0.83 & 0.52 & $1 \cdot 18$ & 0.02 \\
\hline $\begin{array}{l}\text { From bread and cereals } \\
(\mathrm{g} / 1000 \mathrm{~kJ})\end{array}$ & 866 & 0.83 & 0.54 & $1 \cdot 17$ & 0.69 & 0.43 & 0.96 & $<0.0001$ \\
\hline Alcohol (g) & 866 & 1.62 & 0.21 & 9.58 & 1.67 & 0.17 & 9.51 & 0.2 \\
\hline
\end{tabular}

ALT, alanine aminotransferase; GGT, $\gamma$-glutamyltransferase; GI, glycaemic index; GL, glycaemic load.

* Wilcoxon signed-rank test.

† To convert U/l to $\mu$ kat/l, multiply by 0.017 .

intakes of fibre from fruits being the relevant source $(P=0.003$, model 2; Table 4, first main column).

Regarding the associations between carbohydrate quality and HDL-C, a higher dietary GI was cross-sectionally related to lower HDL-C levels ( $P=0 \cdot 046$, model 2 ; Table 4 , third column). A lower starch intake was also related to lower HDL-C levels, but adjustment for confounding factors attenuated this association $(P=0 \cdot 05$, model 2 ; Table 4, third column). For none of the other aspects of carbohydrate nutrition, a relationship to HDL-C could be observed - neither crosssectionally nor concurrently.

\section{Sensitivity analyses}

Additional adjustment for albumin or bilirubin, metabolic variables sometimes also used to assess liver health, did not alter the results. Furthermore, we repeated the analysis for the entire study sample with data on BMES-2 and -3 including also the 113 participants with a habitually high alcohol intake. The results were comparable with those presented for ALT, TAG and HDL-C, with the observed associations being more pronounced. For GGT, both cross-sectional and 5-year concurrent associations were observed with dietary GI and fibre intake (particularly from fruit sources) in the entire study sample (data not shown). To assess any effect modification by liver marker status (e.g. normal or elevated values), we performed stratified analysis which did, however, yield comparable results (data not shown).

\section{Discussion}

To the best of our knowledge, the present study is the first investigating the prospective association of carbohydrate quality with liver enzymes in an older population. The present study confirms previously reported associations between dietary GI and fibre intake with TAG and HDL-C, but suggests that these are not of prospective relevance. Overall, the effect sizes for all observed associations were small. Hence, the present analysis does not support a major role of carbohydrate quality in relation to markers of liver function among the elderly.

Added sugars, particularly from soft drinks, are proposed to be relevant in NAFLD pathophysiology: Kechagias et al. ${ }^{(21)}$ found a positive correlation between the intake of simple sugars and the levels of ALT, and case-control studies have indicated a positive association of NAFLD risk and severity with soft drink consumption ${ }^{(4,5)}$. We did not observe relationships of sugar with any of the analysed markers. This could be due to the fact that soft drinks were rarely consumed by this elderly population. In this context, concern has been expressed that high intakes of fructose primarily derived 
Table 3. Mixed models* of the cross-sectional and the 5-year concurrent relationships of markers of carbohydrate quality to log transformed serum alanine aminotransferase (ALT) and $\gamma$-glutamyltransferase (GGT) levels (U/I) in the 866 Blue Mountains Eye Study (BMES) participants ( $\beta$ Coefficients and standard errors)

\begin{tabular}{|c|c|c|c|c|c|c|c|c|c|c|c|c|}
\hline & \multicolumn{6}{|c|}{ ALT $(n$ 866) } & \multicolumn{6}{|c|}{ GGT $(n 866)$} \\
\hline & \multicolumn{3}{|c|}{$\begin{array}{l}\text { Cross-sectional } \\
\text { estimate }\end{array}$} & \multicolumn{3}{|c|}{$\begin{array}{l}\text { 5-year concurrent } \\
\text { change estimate }\end{array}$} & \multicolumn{3}{|c|}{$\begin{array}{l}\text { Cross-sectional } \\
\text { estimate }\end{array}$} & \multicolumn{3}{|c|}{$\begin{array}{l}5 \text {-year concurrent } \\
\text { change estimate }\end{array}$} \\
\hline & $\beta$ & SE & $P$ & $\beta$ & SE & $P$ & $\beta$ & SE & $P$ & $\beta$ & SE & $P$ \\
\hline \multicolumn{13}{|l|}{$\mathrm{Gl}$} \\
\hline Model 1† & 0.0018 & 0.0039 & 0.6 & 0.0055 & 0.0032 & 0.09 & 0.0068 & 0.0045 & 0.1 & 0.0063 & 0.0035 & 0.07 \\
\hline Model $2 \ddagger$ & 0.0007 & 0.0041 & 0.9 & 0.0043 & 0.0033 & 0.2 & 0.0004 & 0.0048 & 0.9 & 0.0053 & 0.0037 & 0.2 \\
\hline \multicolumn{13}{|c|}{ Sugar intake (\%) } \\
\hline Model $1 \dagger$ & 0.0001 & 0.0025 & 1.0 & 0.0025 & 0.0020 & 0.2 & 0.0046 & 0.0029 & 0.1 & 0.0002 & 0.0022 & 0.9 \\
\hline Model $2 \S$ & 0.0016 & 0.0031 & 0.6 & 0.0017 & 0.0025 & 0.5 & 0.0018 & 0.0036 & 0.6 & 0.0014 & 0.0028 & 0.6 \\
\hline \multicolumn{13}{|c|}{ Starch intake (\%) } \\
\hline Model 1† & 0.0039 & 0.0032 & 0.2 & 0.0021 & 0.0024 & 0.4 & 0.0060 & 0.0037 & 0.1 & 0.0028 & 0.0027 & 0.3 \\
\hline Model $2 \ddagger$ & 0.0008 & 0.0033 & 0.8 & 0.0041 & 0.0026 & 0.1 & 0.0046 & 0.0038 & 0.2 & 0.0033 & 0.0029 & 0.3 \\
\hline \multicolumn{13}{|c|}{ Fibre intake $(\mathrm{g} / 1000 \mathrm{~kJ})$} \\
\hline Model 1† & 0.0032 & 0.0039 & 0.4 & 0.0048 & 0.0034 & 0.2 & 0.0149 & 0.0045 & 0.0009 & 0.0034 & 0.0037 & 0.4 \\
\hline Model 2\| & 0.0004 & 0.0046 & 0.9 & 0.0020 & 0.0038 & 0.6 & 0.0128 & 0.0054 & 0.02 & 0.0020 & 0.0043 & 0.6 \\
\hline \multicolumn{13}{|c|}{$\begin{array}{l}\text { Fibre intake from bread } \\
\text { and cereals }(\mathrm{g} / 1000 \mathrm{~kJ})\end{array}$} \\
\hline Model 1† & 0.0042 & 0.0069 & 0.5 & 0.0115 & 0.0059 & 0.049 & 0.0149 & 0.0080 & 0.06 & 0.0042 & 0.0066 & 0.5 \\
\hline Model 2\| & 0.0020 & 0.0069 & 0.8 & 0.0084 & 0.0060 & 0.2 & 0.0098 & 0.0080 & 0.2 & 0.0052 & 0.0068 & 0.4 \\
\hline \multicolumn{13}{|c|}{$\begin{array}{l}\text { Fibre intake from vegetables } \\
(\mathrm{g} / 1000 \mathrm{~kJ})\end{array}$} \\
\hline Model 1† & 0.0062 & 0.0078 & 0.4 & 0.0052 & 0.0058 & 0.4 & 0.0118 & 0.0091 & 0.2 & 0.0038 & 0.0065 & 0.6 \\
\hline Model 2\| & 0.0043 & 0.0076 & 0.6 & 0.0032 & 0.0058 & 0.6 & 0.0055 & 0.0089 & 0.5 & 0.0066 & 0.0066 & 0.3 \\
\hline \multicolumn{13}{|c|}{$\begin{array}{l}\text { Fibre intake from fruits } \\
(\mathrm{g} / 1000 \mathrm{~kJ})\end{array}$} \\
\hline Model 1† & 0.0032 & 0.0064 & 0.6 & 0.0035 & 0.0055 & 0.5 & 0.0189 & 0.0074 & 0.01 & 0.0079 & 0.0060 & 0.2 \\
\hline Model 2\| & 0.0049 & 0.0081 & 0.5 & 0.0039 & 0.0065 & 0.6 & 0.0158 & 0.0095 & 0.095 & 0.0070 & 0.0073 & 0.3 \\
\hline
\end{tabular}

GI, glycaemic index.

* Models contain a random statement with an unstructured covariance structure.

† Model 1 contains time defined as 1 (BMES-2) and 2 (BMES-3) and the predictor variable (e.g. dietary GI (per 10 units/d)) as terms at baseline, baselinextime and concurrent change, adjustment for sex and energy (use of the multivariate energy density model).

$\ddagger$ Model 1 additionally adjusted for BMl as terms at baseline and baseline $\times$ time, age, diabetes at baseline, smoking (past and/or concurrent), alcohol consumption (categorical) as terms at baseline, baseline $\times$ time and concurrent change, the use of cholesterol-lowering medication and dietary fat (percentage of energy, en\%) and fibre intake ( $\mathrm{g} / \mathrm{MJ}$ ) as terms at baseline, baseline $\times$ time and concurrent change.

$\S$ Same as $\ddagger$, but adjustment for fibre intake from fruits $(\mathrm{g} / \mathrm{MJ})$ as terms at baseline, baseline $\times$ time and concurrent change instead of total fibre intake.

\| Model 1 additionally adjusted for BMI as terms at baseline and baseline $\times$ time, age, diabetes at baseline, smoking (past and/or concurrent), alcohol consumption (categorical) as terms at baseline, baseline $\times$ time and concurrent change, the use of cholesterol-lowering medication and dietary fat intake (en\%) and dietary Gl as terms at baseline, baseline $\times$ time and concurrent change.

from high-fructose maize syrup could adversely affect liver function $^{(22)}$. In contrast to the USA, sucrose is the most commonly used sweetener in Australia, which, however, has been proposed to yield effects comparable with those of high-fructose maize syrup ${ }^{(22)}$. Unfortunately, additional data that would have allowed separate appraisal of intrinsic and added sugar or fructose intake were not available in the present study.

Dietary fibre, particularly viscous fibre, may exert beneficial effects on blood lipids and inflammatory markers ${ }^{(23)}$. Indeed, oxidative stress is increasingly recognised as an important parameter in NAFLD pathophysiology and is another possible mechanism linking carbohydrate quality to hepatic steatosis ${ }^{(24,25)}$. While Valtuena et al. ${ }^{(2)}$ did not observe a relationship between total fibre intake and liver steatosis, we saw a favourable association of dietary fibre with GGT and fasting TAG, in particular for fibre from fruit sources.

In contrast to single nutrients such as fructose or fibre, dietary GI gives an estimate of repeated postprandial glycaemic excursions, i.e. it allows us to address the relevance of one particular metabolic response to carbohydrate nutrition. The assumption that a higher dietary GI could be related to NAFLD stems from the observation that a hyperenergetic intake of carbohydrate-rich foods leading to increased postprandial glucose elevations enhances hepatic lipogenesis $^{(26-28)}$. Valtuena et al. $^{(2)}$ showed an association between dietary GI and the degree of hepatic steatosis measured by liver echography. However, in a stratified analysis, they observed a significant impact of dietary GI only for participants, who were insulin-resistant ( $n$ 60, 24.9\%). They concluded that the combination of hyperglycaemia and hyperinsulinaemia may lead to increased hepatic fat accumulation through elevated lipogenesis, on the one hand, and suppressed $\beta$-oxidation, on the other hand ${ }^{(2)}$. By contrast, we did not observe an interaction with diabetes/impaired fasting glucose status; however, this could reflect insufficient power as the number of participants with these conditions was small ( $n 85,9.8 \%$ ) (data not shown). In the present analysis, dietary GI was not related to liver enzymes. Instead, we observed cross-sectional associations of dietary GI with TAG and HDL-C levels - the latter one also mirrored by a relationship to starch intake, a dietary factor closely related to dietary 
Table 4. Mixed models* of the cross-sectional and the 5-year concurrent relationships of markers of carbohydrate quality to log-transformed serum fasting TAG (mmol/l) and HDL-cholesterol levels (mmol/l) in the 755 Blue Mountains Eye Study (BMES) participants ( $\beta$ Coefficients and standard errors)

\begin{tabular}{|c|c|c|c|c|c|c|c|c|c|c|c|c|}
\hline & \multicolumn{6}{|c|}{ TAG $(n 755)$} & \multicolumn{6}{|c|}{ HDL $(n 755)$} \\
\hline & \multicolumn{3}{|c|}{ Cross-sectional estimate } & \multicolumn{3}{|c|}{$\begin{array}{l}\text { 5-year concurrent change } \\
\text { estimate }\end{array}$} & \multicolumn{3}{|c|}{$\begin{array}{l}\text { Cross-sectional } \\
\text { estimate }\end{array}$} & \multicolumn{3}{|c|}{$\begin{array}{l}\text { 5-year concurrent change } \\
\text { estimate }\end{array}$} \\
\hline & $\beta$ & SE & $P$ & $\beta$ & SE & $P$ & $\beta$ & $\mathrm{SE}$ & $P$ & $\beta$ & SE & $P$ \\
\hline \multicolumn{13}{|l|}{ GI } \\
\hline Model $1 \dagger$ & 0.0123 & 0.0043 & 0.005 & 0.0014 & 0.0035 & 0.7 & 0.0053 & 0.0023 & 0.02 & 0.0009 & 0.0016 & 0.6 \\
\hline Model 2‡ & 0.0066 & 0.0045 & 0.1 & 0.0022 & 0.0037 & 0.6 & 0.0048 & 0.0024 & 0.046 & 0.0021 & 0.0017 & 0.2 \\
\hline \multicolumn{13}{|c|}{ Sugar intake (\%) } \\
\hline Model $1 \dagger$ & 0.0005 & 0.0028 & 0.9 & 0.0008 & 0.0022 & 0.7 & 0.0011 & 0.0015 & 0.5 & 0.0001 & 0.0010 & 0.9 \\
\hline Model $2 \S$ & 0.0028 & 0.0034 & 0.4 & 0.0022 & 0.0028 & 0.4 & 0.0002 & 0.0018 & 0.9 & 0.0011 & 0.0013 & 0.4 \\
\hline \multicolumn{13}{|c|}{ Starch intake (\%) } \\
\hline Model $1 \dagger$ & 0.0045 & 0.0036 & 0.2 & 0.0007 & 0.0027 & 0.8 & 0.0046 & 0.0019 & 0.02 & 0.0020 & 0.0013 & $0 \cdot 1$ \\
\hline Model 2‡ & 0.0044 & 0.0037 & 0.2 & 0.0002 & 0.0029 & 0.9 & 0.0038 & 0.0019 & 0.05 & 0.0020 & 0.0014 & 0.1 \\
\hline \multicolumn{13}{|c|}{ Fibre intake $(\mathrm{g} / 1000 \mathrm{~kJ})$} \\
\hline Model $1 \dagger$ & 0.0135 & 0.0043 & 0.002 & 0.0030 & 0.0038 & 0.4 & 0.0027 & 0.0023 & 0.2 & 0.0028 & 0.0018 & 0.1 \\
\hline Model 2\| & 0.0161 & 0.0051 & 0.002 & 0.0038 & 0.0044 & 0.4 & 0.0037 & 0.0027 & 0.2 & 0.0036 & 0.0020 & 0.08 \\
\hline \multicolumn{13}{|c|}{$\begin{array}{l}\text { Fibre intake from bread } \\
\text { and cereals }(\mathrm{g} / 1000 \mathrm{~kJ})\end{array}$} \\
\hline Model 1† & 0.0108 & 0.0076 & 0.2 & 0.0103 & 0.0066 & 0.1 & 0.0039 & 0.0040 & 0.3 & 0.0030 & 0.0031 & 0.3 \\
\hline Model 2\| & 0.0108 & 0.0076 & 0.2 & 0.0117 & 0.0068 & 0.09 & 0.0047 & 0.0040 & 0.2 & 0.0034 & 0.0032 & 0.3 \\
\hline \multicolumn{13}{|c|}{$\begin{array}{l}\text { Fibre intake from vegetables } \\
(\mathrm{g} / 1000 \mathrm{~kJ})\end{array}$} \\
\hline Model $1 \dagger$ & 0.0095 & 0.0087 & 0.3 & 0.0057 & 0.0066 & 0.4 & 0.0023 & 0.0046 & 0.6 & 0.0017 & 0.0031 & 0.6 \\
\hline Model 2\| & 0.0087 & 0.0084 & 0.3 & 0.0059 & 0.0066 & 0.4 & 0.0033 & 0.0044 & 0.5 & 0.0008 & 0.0031 & 0.8 \\
\hline \multicolumn{13}{|c|}{$\begin{array}{l}\text { Fibre intake from fruits } \\
(\mathrm{g} / 1000 \mathrm{~kJ})\end{array}$} \\
\hline Model $1 \dagger$ & 0.0225 & 0.0070 & 0.001 & 0.0068 & 0.0061 & 0.3 & 0.0043 & 0.0037 & 0.3 & 0.0041 & 0.0028 & 0.1 \\
\hline Model 2\| & 0.0269 & 0.0089 & 0.003 & 0.0102 & 0.0072 & 0.2 & 0.0046 & 0.0047 & 0.3 & 0.0058 & 0.0034 & 0.09 \\
\hline
\end{tabular}

GI, glycaemic index.

${ }^{*}$ Models contain a random statement with an unstructured covariance structure.

† Model 1 contains time defined as 1 (BMES-2) and 2 (BMES-3) and the predictor variable (e.g. dietary GI per 10 units/d) as terms at baseline, baselinextime and concurrent change, adjustment for sex and energy (use of the multivariate energy density model).

¥Model 1 additionally adjusted for BMI as terms at baseline and baseline $\times$ time, age, diabetes at baseline, smoking (past and/or concurrent), alcohol consumption (categorical) as terms at baseline, baseline $\times$ time and concurrent change, the use of cholesterol-lowering medication and dietary fat (percentage of energy, en\%) and fibre intake ( $\mathrm{g} / \mathrm{MJ}$ ) as terms at baseline, baseline $\times$ time and concurrent change.

$\S$ Same as $\ddagger$, but adjustment for fibre intake from fruits $(\mathrm{g} / \mathrm{MJ})$ as terms at baseline, baseline $\times$ time and concurrent change instead of total fibre intake.

\| Model 1 additionally adjusted for BMI as terms at baseline and baseline $\times$ time, age, diabetes at baseline, smoking (past and/or concurrent), alcohol consumption (categorical) as terms at baseline, baseline $\times$ time and concurrent change, the use of cholesterol-lowering medication and dietary fat intake (en\%) and dietary GI as terms at baseline, baseline $\times$ time and concurrent change.

GI values ${ }^{(29)}$. The present findings are in line with other crosssectional observational studies ${ }^{(30-36)}$; however, the absence of a longitudinal relationship questions the clinical relevance of these associations in this age group. Furthermore, evidence from meta-analyses of intervention studies does not support an effect of dietary GI on TAG or HDL-C ${ }^{(37,38)}$.

The main strengths of the present analysis were the prospective study design with repeated measurements of liver enzymes, fasting TAG and HDL-C, as well as dietary intake data in a contemporary sample of older men and women. We could control for repeatedly measured key confounding factors such as BMI, medications taken or health status. However, we cannot preclude residual confounding, resulting from imprecisely measured or unmeasured confounding factors. Additionally, due to the large number of tests, chance findings are a possibility.

Regarding the markers for NAFLD, it has to be considered that raised liver enzymes can only reveal an increased risk, yet no quantitative conclusions about the extent of hepatic fat accumulation can be drawn. Moreover, NAFLD can be present without any elevations in liver enzymes. However, these markers are commonly used as minimally invasive parameters $^{(1,8)}$, and were readily available for the present study sample. Despite the fact that we employed a validated FFQ, misclassification bias can still exist. This may apply to fibre sources in particular, since the FFQ was neither designed nor validated for fibre source-specific analyses. At the 5-year follow-up, participants may have had a greater recall of certain food groups increasingly considered healthy such as fruits and vegetables. However, such a recall bias would have rather translated into an underestimation for the concurrent changes in markers of liver function. Selection bias resulting from the limited number of persons eligible for the longitudinal analysis is a particular concern and may hamper the extent to which the present results can be generalised. Similar characteristics among those lost to follow-up suggest that attrition bias introduced by loss to follow-up was low. However, attrition due to higher natural mortality limits the generalisability of the present findings. Also, selective survival may have occurred because those included in the analysis were healthier at baseline regarding some but not all clinical parameters than those who died during the follow-up. 
In conclusion, the absence of longitudinal associations between carbohydrate quality and liver enzymes or serum lipids in this older Australian population does not support a major role of carbohydrate nutrition in liver function among the elderly. A potential impact of carbohydrate nutrition in populations with more adverse dietary habits deserves further investigation, ideally using direct measures of NAFLD status

\section{Acknowledgements}

The BMES was supported by the Australian National Health and Medical Research Council. P. M. and A. E. B. conceived the project; P. M., B. G. and E. R. collected the data; B. G., E. R. and G. C. provided the databases and/or statistical experience, J. G. analysed the data and wrote the manuscript; A. E. B. supervised the project; B. G., E. R., A. W. B., G. C., J. C. B.-M. and P. M. critically revised the manuscript for important intellectual content. All authors read and approved the final version of the paper. Conflicts of interest: J. C. B.-M. is the director of a notfor-profit GI-based food endorsement programme in Australia and manages the Sydney University Glycaemic Index Research Service; A. W. B. is a consultant for Glycaemic Index Limited, a not-for-profit GI-based food endorsement programme in Australia; J. G., A. E. B., B. G., E. R., G. C. and P. M. have no conflicts of interest.

\section{References}

1. Stefan N, Kantartzis K \& Haring HU (2008) Causes and metabolic consequences of fatty liver. Endocr Rev 29, 939-960.

2. Valtuena S, Pellegrini N, Ardigo D, et al. (2006) Dietary glycemic index and liver steatosis. Am J Clin Nutr $\mathbf{8 4}$, 136-142 (quiz 268-139).

3. Fraser A, Abel R, Lawlor DA, et al. (2008) A modified Mediterranean diet is associated with the greatest reduction in alanine aminotransferase levels in obese type 2 diabetes patients: results of a quasi-randomised controlled trial. Diabetologia 51, 1616-1622.

4. Assy N, Nasser G, Kamayse I, et al. (2008) Soft drink consumption linked with fatty liver in the absence of traditional risk factors. Can J Gastroenterol 22, 811-816.

5. Zelber-Sagi S, Nitzan-Kaluski D, Goldsmith R, et al. (2007) Long term nutritional intake and the risk for non-alcoholic fatty liver disease (NAFLD): a population based study. $J$ Hepatol 47, 711-717.

6. Toshimitsu K, Matsuura B, Ohkubo I, et al. (2007) Dietary habits and nutrient intake in non-alcoholic steatohepatitis. Nutrition 23, 46-52.

7. Musso G, Gambino R, De Michieli F, et al. (2003) Dietary habits and their relations to insulin resistance and postprandial lipemia in nonalcoholic steatohepatitis. Hepatology 37, 909-916.

8. Angulo P (2002) Nonalcoholic fatty liver disease. $N$ Engl J Med 346, 1221-1231.

9. Westerbacka J, Corner A, Tiikkainen M, et al. (2004) Women and men have similar amounts of liver and intraabdominal fat, despite more subcutaneous fat in women: implications for sex differences in markers of cardiovascular risk. Diabetologia 47, 1360-1369.
10. Paschos P \& Paletas K (2009) Non alcoholic fatty liver disease and metabolic syndrome. Hippokratia 13, 9-19.

11. Bacon BR, Farahvash MJ, Janney CG, et al. (1994) Nonalcoholic steatohepatitis: an expanded clinical entity. Gastroenterology 107, 1103-1109.

12. Diehl AM, Goodman Z \& Ishak KG (1988) Alcohollike liver disease in nonalcoholics. A clinical and histologic comparison with alcohol-induced liver injury. Gastroenterology $\mathbf{9 5}$, 1056-1062.

13. Vanni E, Bugianesi E, Kotronen A, et al. (2010) From the metabolic syndrome to NAFLD or vice versa? Dig Liver Dis 42, 320-330.

14. Kim HJ, Lee KE, Kim DJ, et al. (2004) Metabolic significance of nonalcoholic fatty liver disease in nonobese, nondiabetic adults. Arch Intern Med 164, 2169-2175.

15. Buyken AE, Flood V, Rochtchina E, et al. (2010) Modifications in dietary fat quality are associated with changes in serum lipids of older adults independently of lipid medication. J Nutr 140, 88-94.

16. Smith W, Mitchell P, Reay EM, et al. (1998) Validity and reproducibility of a self-administered food frequency questionnaire in older people. Aust $N Z J$ Public Health 22, 456-463.

17. Willett WC, Sampson L, Browne ML, et al. (1988) The use of a self-administered questionnaire to assess diet four years in the past. Am J Epidemiol 127, 188-199.

18. Barclay AW, Flood VM, Brand-Miller JC, et al (2008) Validity of carbohydrate, glycaemic index and glycaemic load data obtained using a semi-quantitative food-frequency questionnaire. Public Health Nutr 11, 573-580.

19. Foster-Powell K, Holt SH \& Brand-Miller JC (2002) International table of glycemic index and glycemic load values: 2002. Am J Clin Nutr 76, 5-56.

20. Hu FB, Stampfer MJ, Rimm E, et al. (1999) Dietary fat and coronary heart disease: a comparison of approaches for adjusting for total energy intake and modeling repeated dietary measurements. Am J Epidemiol 149, 531-540.

21. Kechagias S, Ernersson A, Dahlqvist O, et al. (2008) Fast-food-based hyper-alimentation can induce rapid and profound elevation of serum alanine aminotransferase in healthy subjects. Gut 57, 649-654.

22. Stanhope KL (2011) Role of fructose-containing sugars in the epidemics of obesity and metabolic syndrome. Annu Rev Med 63, 329-343.

23. Galisteo M, Duarte J \& Zarzuelo A (2008) Effects of dietary fibers on disturbances clustered in the metabolic syndrome. J Nutr Biochem 19, 71-84.

24. Dey A \& Swaminathan K (2010) Hyperglycemia-induced mitochondrial alterations in liver. Life Sci $\mathbf{8 7}, 197-214$.

25. Hu Y, Block G, Norkus EP, et al. (2006) Relations of glycemic index and glycemic load with plasma oxidative stress markers. Am J Clin Nutr 84, 70-76 (quiz 266-267).

26. Timlin MT, Barrows BR \& Parks EJ (2005) Increased dietary substrate delivery alters hepatic fatty acid recycling in healthy men. Diabetes 54, 2694-2701.

27. Hudgins LC, Seidman CE, Diakun J, et al. (1998) Human fatty acid synthesis is reduced after the substitution of dietary starch for sugar. Am J Clin Nutr 67, 631-639.

28. Schwarz JM, Linfoot P, Dare D, et al. (2003) Hepatic de novo lipogenesis in normoinsulinemic and hyperinsulinemic subjects consuming high-fat, low-carbohydrate and low-fat, high-carbohydrate isoenergetic diets. Am J Clin Nutr 77, 43-50.

29. Atkinson FS, Foster-Powell K \& Brand-Miller JC (2008) International tables of glycemic index and glycemic load values: 2008. Diabetes Care 31, 2281-2283. 
30. Frost G, Leeds AA, Dore CJ, et al. (1999) Glycaemic index as a determinant of serum HDL-cholesterol concentration. Lancet 353, 1045-1048.

31. Liu S, Manson JE, Stampfer MJ, et al. (2001) Dietary glycemic load assessed by food-frequency questionnaire in relation to plasma high-density-lipoprotein cholesterol and fasting plasma triacylglycerols in postmenopausal women. $A m \mathrm{~J}$ Clin Nutr 73, 560-566.

32. Mosdol A, Witte DR, Frost G, et al. (2007) Dietary glycemic index and glycemic load are associated with high-density-lipoprotein cholesterol at baseline but not with increased risk of diabetes in the Whitehall II study. Am J Clin Nutr 86, 988-994.

33. Ford ES \& Liu S (2001) Glycemic index and serum high-density lipoprotein cholesterol concentration among us adults. Arch Intern Med 161, 572-576.

34. Murakami K, Sasaki S, Takahashi Y, et al. (2006) Dietary glycemic index and load in relation to metabolic risk factors in Japanese female farmers with traditional dietary habits. Am J Clin Nutr 83, 1161-1169.

35. Ma Y, Li Y, Chiriboga DE, et al. (2006) Association between carbohydrate intake and serum lipids. J Am Coll Nutr 25, 155-163.

36. Du H, van der AD, van Bakel MM, et al. (2008) Glycemic index and glycemic load in relation to food and nutrient intake and metabolic risk factors in a Dutch population. Am J Clin Nutr 87, 655-661.

37. Kelly SAM, Frost G, Whittaker V, et al. (2004) Low glycaemic index diets for coronary heart disease. The Cochrane Database of Systematic Reviews 2004, issue 4, CD004467.

38. Goff LM, Cowland DE, Hooper L, et al. (2012) Low glycemic index diets and blood lipids: a systematic review and meta-analysis of randomised controlled trials. Nutr Metab Cardiovasc Dis (epublication ahead of print version 25 July 2012). 